\title{
Quantifying the large-scale electrification equilibrium effects in dust storms using field observations at Qingtu Lake Observatory
}

\author{
Huan Zhang ${ }^{1}$ and Xiaojing Zheng ${ }^{1,2}$ \\ ${ }^{1}$ Department of Mechanics, Lanzhou University, Key Laboratory of Mechanics on Disaster and Environment in Western \\ China, The Ministry of Education of China, Lanzhou 730000, China \\ ${ }^{2}$ Research Center for Applied Mechanics, School of Mechano-Electronic Engineering, Xidian University, \\ Xi'an 710071, China
}

Correspondence: Xiaojing Zheng (xjzheng@xidian.edu.cn)

Received: 19 March 2018 - Discussion started: 26 June 2018

Revised: 19 October 2018 - Accepted: 12 November 2018 - Published: 4 December 2018

\begin{abstract}
Dust and sand electrification, which is a ubiquitous phenomenon in dust events, has a potentially dramatic effect on dust and sand lifting and transport processes. However, the effect of such electrification is still largely unclear, mainly due to its complexity and sparse observations. Here, we conducted an extensive observational analysis involving mild and severe dust storms with minimum visibility, ranging from $\sim 0.09$ to $0.93 \mathrm{~km}$, to assess the electrical properties of airborne dust particles in dust storms. The space charge density has been estimated indirectly based on Gauss's law. Using the wavelet coherence analysis that is a method for evaluating the correlations between two non-stationary time series in the time-frequency domain, we found that the space charge density and dust concentration were significantly correlated over the $10 \mathrm{~min}$ timescales (on the order of the typical integral timescale of atmospheric turbulence). We further presented a simple linear regression (SLR) model to quantify such large timescale correlations and found that there was a significant linear relationship between space charge density and dust concentration at given ambient temperature and relative humidity $(\mathrm{RH})$, suggesting that the estimated mean charge-to-mass ratio of dust particles was expected to remain constant (termed as the equilibrium value $\mu^{*}$ ). In addition, the influences of ambient temperature and $\mathrm{RH}$ on $\mu^{*}$ were evaluated by a multiple linear regression (MLR) model, showing that the $\mu^{*}$ is nonlinearly related to environmental factors. The present study provides observational evidence for the environmental-dependent electrification equilibrium effects in dust storms. This finding may reduce challenges in future quantifications of dust electrification, as it is possible
\end{abstract}

to exclude effects, such as the particles' collisional dynamics, on dust electrification.

\section{Introduction}

Granular materials in dust events such as blowing sand, dust devils and dust storms, are frequently brought into contact or collision with each other, accumulating large amounts of electrical charge on their surfaces (Kamra, 1972; Rudge, 1913; Schmidt et al., 1998). The strong electrostatic forces exerted on dust particles, which are comparable to gravitational force, could considerably affect the motion of particles and facilitate the lifting of particles from the ground (Esposito et al., 2016; Harper et al., 2017; Kok and Renno, 2008; Schmidt et al., 1998; Zheng et al., 2003). The first mutual coupling numerical saltation model (that is, wind flow, particle motion and electrostatic forces interacting with each other), developed by Zheng et al. (2003), showed that vertically downward-pointing electrostatic forces strongly lowered particle trajectories (and conversely, upward-pointing forces elevated particle trajectories). Additionally, recent observations have demonstrated that electrostatic forces can enhance dust concentration by up to a factor of 10 when the intensity of the electric field (E-field) exceeds a certain threshold value (Esposito et al., 2016). In addition to affecting dust transport, the E-fields created by charged dust may contribute to detection and early warning of severe dust events. For example, Aizawa et al. (2016) and Zhang et al. (2017) have documented that the substantial enhancement in the inten- 
sity of E-field occurs prior to the arrival of the dust clouds (fronts). Therefore, there is the considerable impetus to investigate electrical effects in dust events.

Measurements of electrical effects in dust events (especially dust storms) have been made for over 100 years, dating back to 1913 with the finding that the atmospheric vertical E-fields increased by 1-3 orders of magnitude and reversed their direction during dust storms (Rudge, 1913; Schmidt et al., 1998; Williams et al., 2009). However, owing to the difficulty in monitoring random dust events, only several tens of events are recorded in the literature (see Table 3 in Zheng, 2013, for details). Previous studies are mainly concerned with the vertical profile of E-fields in the near-ground region and their influence on dust transport (e.g., Esposito et al., 2016; Kamra, 1972; Rudge, 1913; Yair et al., 2016). The measurements showed that the vertical E-fields decrease with increasing height in the sub-meter region (e.g., Schmidt et al., 1998), but exhibit multi-layer properties in the region of up to $30 \mathrm{~m}$ height (Zhang et al., 2017). Though measurements of E-fields in dust events are numerous, the measurements associated with the electrical properties of dust particles are relatively sparse. So far, only the charge-to-mass ratio of saltating particles (in the range of $<10 \mathrm{~cm}$ height) has been measured using a Faraday cage, showing that its magnitude is on the order of $\sim 60 \mu \mathrm{C} \mathrm{kg}^{-1}$ (Bo et al., 2014; Schmidt et al., 1998). To better understand the electrical properties of airborne dust particles, further measurements are therefore required.

Furthermore, a large number of theoretical works and laboratory experiments have shown that the net electrical charge on millimeter-sized particles increases with increasing number of collisions/contacts and correlates with the particles' kinetic energy (Apodaca et al., 2010; Harper and Dufek, 2016; Matsuyama and Yamamoto, 1995; Zhang et al., 2013) until particles acquired a certain amount of charge (termed as equilibrium charge). The magnitude of the eventual equilibrium charge on particles is found to be independent of the particles' collisional dynamics and therefore will reduce the difficulties in the quantification of particle electrification. However, whether such an electrification equilibrium exists under natural circumstances, especially for micron-sized dust particles in dust storms, is unclear and needs to be verified. Additionally, preliminary research (Esposito et al., 2016; Merrison, 2012; Xie and Han, 2012; Zhang et al., 2017; Zheng et al., 2014) suggests that environmental factors such as the ambient temperature and RH could considerably affect the intensity of E-fields. It is still unknown whether environmental factors have an impact on the properties of dust electrification, and especially electrification equilibrium effects in a dust storm.

The ratio of space charge density to the dust mass concentration (called mean charge-to-mass ratio of dust particles) rather than charge on the individual particles is generally used to quantify the electrical properties of dusty phenomena. In this study, we build on a set of field observations through an extensive statistical analysis to assess the mean scaled charge-to-mass ratio of airborne dust particles $\mu^{*}$ (defined in Sect. 2.2) in dust storms and to untangle the influences of environmental factors (i.e., ambient temperature and $\mathrm{RH})$ on the $\mu^{*}$. Therefore, an electrification equilibrium is said to be built if $\mu^{*}$ remains constant at the given ambient temperature and RH. Similar to the indirect method for determining the space charge density of thunderstorms, clouds and aerosol layers in the atmosphere (e.g., MacGorman and Rust, 1998; Nicoll, 2012; Stolzenburg and Marshall, 1994; Zhou and Tinsley, 2007), we estimated the multi-meter averaged space charge density based on Gauss's law, in which the mean space charge density is proportional to the divergence of the E-fields, as described in detail in Sect. 2.2. We evaluated correlations between the space charge density and the dust concentration in time and frequency space through wavelet coherence analysis. We then used an SLR model to assess whether the electrification equilibrium effects exist in dust storms. Finally, we developed an MLR model to evaluate the effects of ambient temperature and $\mathrm{RH}$ on the equilibrium charge.

\section{Methods}

\subsection{Description of the field site}

We conducted field observations in a flat-bottomed dry lakebed of the Qingtu Lake (approximately $39^{\circ} 12^{\prime} 27^{\prime \prime} \mathrm{N}$, $103^{\circ} 40^{\prime} 03^{\prime \prime} \mathrm{E}$ ), approximately $90 \mathrm{~km}$ northeast of Minqin, Gansu, China (Fig. 1a), over the period from 21 March to 2 June 2017. The area was selected since it lies within a dusty belt in the Hexi Corridor (Wang et al., 2018), which is mainly affected by the Mongolian cyclones (and probably by the cold downdrafts from thunderstorms/squall) during the observational period and is therefore frequently subjected to dust events (Shao, 2000; Williams et al., 2009). At the Qingtu Lake Observational Array (QLOA) site, there is a prevailing wind direction, along with a straight line having a mean angle of $\sim 247.5^{\circ}$ with respect to the north (approximately $74.6 \%$ frequency), as shown in Fig. S1 in the Supplement. Measurements of the size distribution of airborne dust particles (Fig. S2) and saltating particles (Fig. S3) implies that the dust events occurring in the QLOA site have a very similar particle size distribution. The QLOA site consists of 21 observation towers distributed in two mutually perpendicular directions (the prevailing wind direction and the spanwise direction). Among these towers, the main tower with a $32 \mathrm{~m}$ height could be used to measure the vertical E-field gradients, and the remaining 20 towers with $5 \mathrm{~m}$ height could be designed to determine the streamwise and spanwise gradients of E-fields (Fig. 1b). 


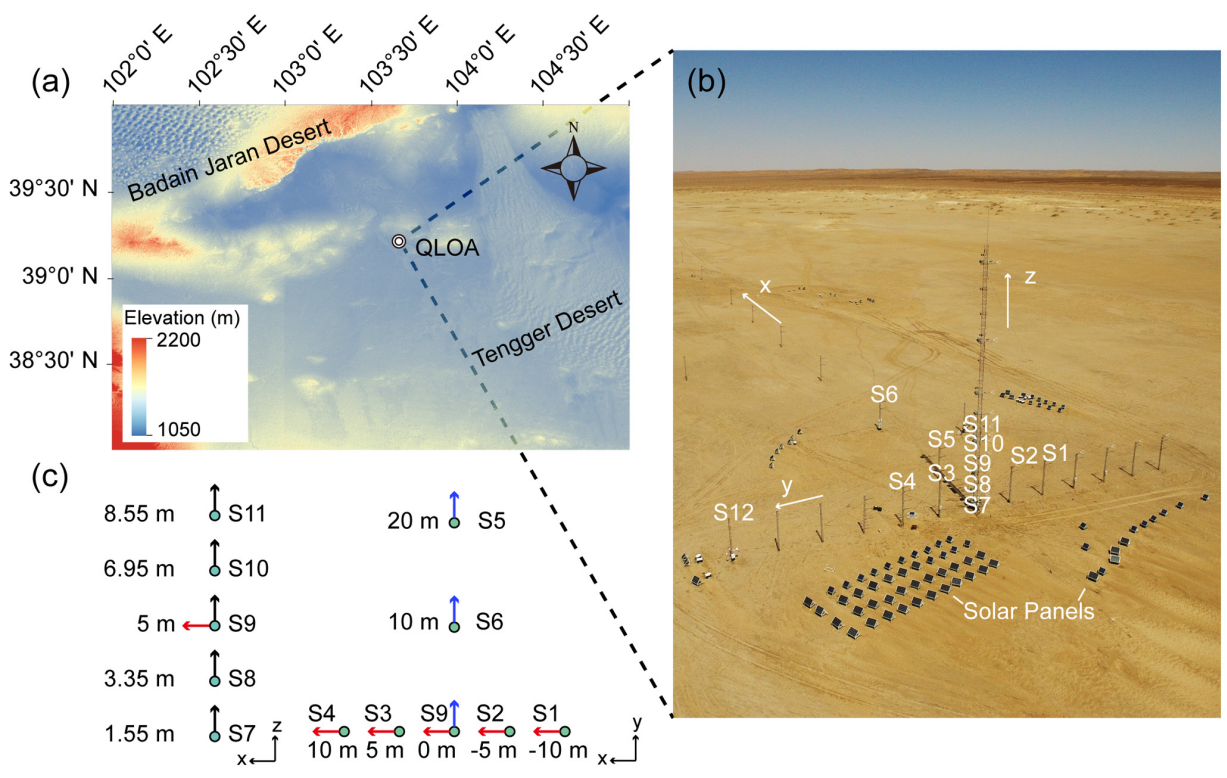

Figure 1. Location of the field site and the observational set-up. (a) The QLOA site is located between the Badain Jaran Desert and the Tengger Desert (a dusty belt that has high-frequency dust events). (b) E-fields were measured at sites S1-S11; 3-D wind velocity was measured at the centrally located S9. The ambient temperature, RH, saltating sand number density and visibility were measured at S12. All of the instruments were powered by a solar panel system. The $x, y$ and $z$ axis are parallel to the prevailing wind direction, spanwise direction and vertical direction, respectively. (c) Layout of VREFMs (not to scale); that is, blue, red and dark arrows correspond to the $x, y$ and $z$ component measurements of E-fields, respectively. It is worth noting that the $x$ and $y$ components of E-fields are generally non-zero because dust transport is non-uniform in the horizontal plane (Zheng, 2013).

\subsection{Measurements}

In dust storms, space charge density $\rho$ can be expressed as the product of the mean charge-to-mass ratio of dust particles $\mu$ and the total dust mass concentration $M_{10} / \lambda$, where $M_{10}$ and $\lambda$ denote the $\mathrm{PM}_{10}$ mass concentration and the $\mathrm{PM}_{10}$ mass fraction, respectively. As mentioned in Sect. 2.1, the size distribution of airborne dust particles varies slightly from event to event. Consequently, the scaled mean charge-to-mass ratio $\mu^{*}$, which is a common measure of the charge-to-mass ratio of dust particles, can be defined as

$\mu^{*} \equiv \frac{\rho}{M_{10}}$,

where $\mu^{*}=\mu / \lambda$ ( $\lambda$ is assumed to be a constant) is equal to the mean charge-to-mass ratio divided by the $\mathrm{PM}_{10}$ mass fraction. From this definition, $\mu^{*}$ can be determined once the space charge density and $\mathrm{PM}_{10}$ mass concentration have been determined. By adopting the International System of Units (SI), the units of $\mu^{*}$ and $\mu$ are $\mathrm{C} \mathrm{kg}^{-1}$, the unit of $\rho$ is $\mathrm{C} \mathrm{m}^{-3}$, the unit of $\mathrm{PM}_{10}$ concentration $M_{10}$ is $\mathrm{kg} \mathrm{m}^{-3}$, and the $\mathrm{PM}_{10}$ mass fraction $\lambda$ has a dimensional unit in Eq. (1).

In the 2017 field campaign, the indirect method mentioned in the introduction was used to estimate the $\mu^{*}$ at a site $5 \mathrm{~m}$ height above the ground (i.e., S9). A total of 11 measurement sites (S1-S11 distributed on 7 observation towers) were arranged to estimate the space charge density at $S 9$, as shown in Fig. $1 \mathrm{~b}$ and c. In theory, the space charge density $\rho$ at $\mathrm{S} 9$, which can directly reflect the polarity of the dust cloud at this height, is related to the corresponding divergence of the E-fields $\nabla \cdot E$ by Gauss's Law (Pollack and Stump, 2002)

$\rho=\varepsilon_{0} \frac{\partial E_{i}}{\partial x_{i}}$,

where $\varepsilon_{0}=8.854 \times 10^{-12} \mathrm{C}^{2} \mathrm{~N}^{-1} \mathrm{~m}^{-2}$ is the permittivity constant in a vacuum; suffix $i$ is summed from 1 to 3 by making use of the Einstein summation convention. In Eq. (2), to determine the partial derivatives of E-fields $E_{i}$ with respect to $x_{i}$ at $\mathrm{S} 9$, E-fields sensors called vibrating-reed electric field mill (VREFM), which were developed by Lanzhou University (Zheng, 2013), were installed in the streamwise, spanwise and wall-normal monitoring networks (Fig. 1c). The VREFM functions were based on measuring the induced electrical charge and were calibrated by a large parallelplate E-field calibrator ( $1 \mathrm{~m}^{2}$ plates). More detailed information of the VREFM is given in our previous works (see Zhang et al., 2017; Zheng, 2013). The partial derivatives of E-fields were estimated from the interpolation-based numerical method and will be shown to be both positive and negative (see Fig. S5 in the Supplement).

The $\mathrm{PM}_{10}$ mass concentration at $\mathrm{S} 9$ was measured by a DustTrak II Aerosol Monitor (Model 8530EP, TSI Incorporated). Additionally, to explore the effects of environmental factors on $\mu^{*}$, additional instruments installed at S12 in- 
clude: sonic anemometer (CSAT3B, Campbell Scientific), measuring three-dimensional (3-D) wind velocity; a sand particle counter (SPC-91, Niigata Electric Co., Ltd.), measuring saltating sand particle number density in the range of $\sim 30-490 \mu \mathrm{m}$ with 32 bins; a temperature-humidity sensor (Model 41003, R. M. Young Company), measuring ambient temperature and RH; and visibility sensor (Model 6000, Belfort Instrument), measuring visibility ranging from $5 \mathrm{~m}$ to $10 \mathrm{~km}$ with $\pm 10 \%$ accuracy (Fig. S4 in the Supplement). Before performing field measurements, all instruments were carefully calibrated in the laboratory. The VREFM sensors were also calibrated at QLOA site by comparing its output to a higher accuracy atmospheric E-field mill (see Fig. S8 in the Supplement). To achieve the best possible instrument accuracy, we performed re-calibration for VREFM sensors and periodic cleaning for Aerosol Monitor 8530EP twice a month during the observational period. All instruments were monitored continuously and simultaneously with a sampling frequency of $1 \mathrm{~Hz}$ (except for the CSAT3B which had a sampling frequency of $50 \mathrm{~Hz}$ ).

\subsection{Wavelet coherence analysis}

For two stationary time series, the ensemble-mean values are equal to the time-mean values; thus, the cross-correlation function and cross-spectral density function are respectively used to measure the joint statistical properties in the time and frequency domain (Bendat and Piersol, 2011). In practice, however, the geophysical time series is generally nonstationary (see Fig. 2) and therefore consists of time-varying statistical properties (Grinsted et al., 2004; Holman et al., 2011). In this study, wavelet coherence analysis is used to assess the correlations between the space charge density $\rho$ and the $\mathrm{PM}_{10}$ dust concentration $M_{10}$ in the time-frequency domain. For two time series, $X$ and $Y$, the squared wavelet coherence is defined as (Grinsted et al., 2004)

$$
R^{2}(n, s)=\frac{\left|\left\langle s^{-1} W^{X Y}(n, s)\right\rangle\right|^{2}}{\left|\left\langle s^{-1} W^{X}(n, s)\right\rangle\right|^{2}\left|\left\langle s^{-1} W^{Y}(n, s)\right\rangle\right|^{2}}
$$

where the brackets \langle\rangle are the smoothing operator in time and scale; $W^{X Y}=W^{X} W^{Y *}$ is the cross wavelet transform; and superscript ${ }^{*}$ is the complex conjugation. The Morlet wavelet (with $\omega_{0}=6$ ) is used to perform the continuous wavelet transforms $W^{X}$ and $W^{Y}$. The squared wavelet coherence $R^{2}(n, s)$ ranges from 0 to 1 and can be thought of as a localized correlation coefficient between two time series in time and frequency space (Grinsted et al., 2004; Holman et al., 2011; Wang et al., 2017). A Matlab toolbox for wavelet coherence analysis can be found at http://noc.ac.uk/ using-science/crosswavelet-wavelet-coherence (last access: 21 November 2018; Grinsted et al., 2004; Wang et al., 2017).

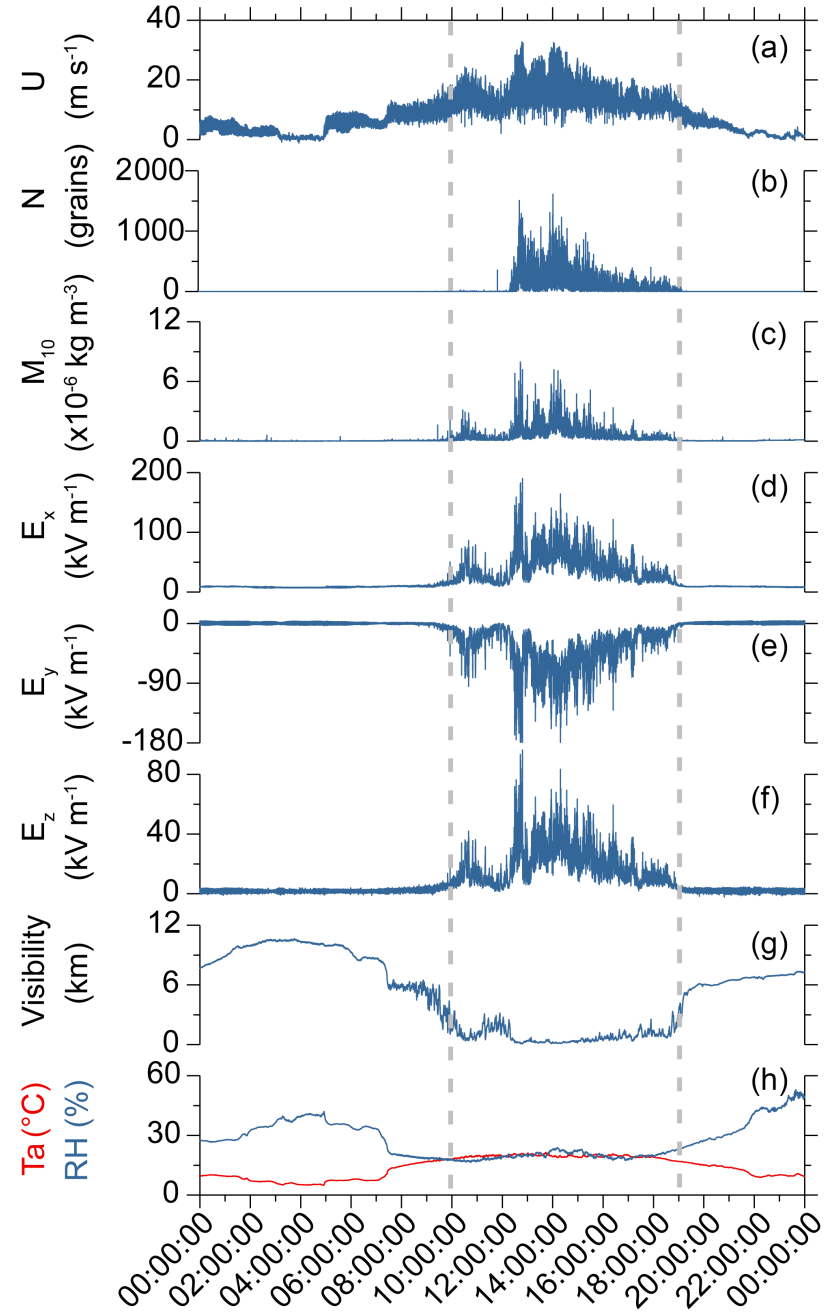

Figure 2. Data series recorded on 17 April 2017 (UTC+8). Topto-bottom: streamwise wind speed, saltating sand number passing through the measurement area ( $2 \mathrm{~mm}$ in height and $25 \mathrm{~mm}$ in length) per second, $\mathrm{PM}_{10}$ mass concentration and 3-D E-fields at S9; visibility, ambient temperature and $\mathrm{RH}$ at $\mathrm{S} 12$. The E-fields $E_{x}, E_{y}$ and $E_{z}$ are positive if they point in the positive directions of $x, y$ and $z$ axes depicted in Fig. 1. That is, $E_{z}$ and fair-weather atmospheric $E$-field are oppositely directed.

\subsection{Regression analysis}

An SLR model was used to explore whether the electrification equilibrium effects exist in dust storms. Theoretically, there is an electrification equilibrium effect (i.e., $\mu^{*}$ is constant) if $\rho$ and $M_{10}$ are linearly correlated at a given ambient temperature and RH (and obviously, nonlinear relationship between them means time-varying $\mu^{*}$ ). Since previous studies (Esposito et al., 2016; Harper and Dufek, 2016; Merrison, 2012; Wiles et al., 2007; Xie and Han, 2012; Zhang et al., 2017; Zheng et al., 2014) suggested that environmental factors such as ambient temperature and RH could considerably affect the intensity of E-fields, SLRs in this study were 
performed at a set of given temperature and $\mathrm{RH}$ intervals of length $2{ }^{\circ} \mathrm{C}$ and $2 \%$, respectively. Although theoretical consideration suggests that the intercept of the linear model (Eq. 1) should be zero, it is also present due to the inaccuracy of measurements and the requirement of statistical inference procedures for linear models. Thus, the SLR model has the form

$\rho=a_{0}+a_{1} M_{10}+\varepsilon_{1}$,

where coefficient $a_{0}$ is the fitted intercept, the fitted slope $a_{1}$ is equal to $\mu^{*}$, and $\varepsilon_{1}$ is a disturbance term. We use the ordinary least squares method to estimate the coefficients of the SLR model (Eq. 4). The $F$ test is used for testing the SLR model.

Previous studies have shown that charge transfer processes are nonlinearly related to the ambient temperature and $\mathrm{RH}$ (Gu et al., 2013; Lacks and Sankaran, 2011; McCarty and Whitesides, 2008; Wei and Gu, 2015; Zheng et al., 2014). We thus use the following MLR to evaluate the effects of ambient temperature and $\mathrm{RH}$ on $\mu^{*}$ :

$\mu^{*}=b_{0}+b_{1} T_{\mathrm{a}}+b_{2} \mathrm{RH}+b_{3} T_{\mathrm{a}}^{2}+b_{4} \mathrm{RH}^{2}+b_{5} T_{\mathrm{a}} \mathrm{RH}+\varepsilon_{2}$,

where $T_{\mathrm{a}}$ and $\mathrm{RH}$ are the ambient temperature and $\mathrm{RH}$, respectively; $b_{i}(i=0,1, \ldots, 5)$ is the fitted coefficients; and $\varepsilon_{2}$ is a disturbance term. The multiple regression analyses were undertaken with $R$ (v.3.4.1).

\section{Results}

\subsection{Electrification equilibrium effects over large timescales}

Fortunately, we have successfully recorded 10 dust storms lasting a total of $\sim 66 \mathrm{~h}$, which is sufficient to perform a series of reliable statistical analyses (Table 1). As an example, Fig. 2 shows the complete data series recorded during a severe dust storm (Movie S1) with the maximum streamwise E-field intensity of up to $\sim 181 \mathrm{kV} \mathrm{m}^{-1}$ (much less than the dielectric strength of air), suggesting that dust particles are highly electrified.

As shown in Fig. 3, on timescales of less than 2 min, there is very low wavelet coherence between $\rho$ and $M_{10}$ throughout the whole period of dust storms, suggesting that the highfrequency fluctuation of dust concentration (probably due to turbulence) is not associated with the space charge density. In contrast, $\rho$ and $M_{10}$ are significantly in-phase correlated on timescales longer than $\sim 10 \mathrm{~min}$ (with coherence power of up to $\sim 0.9-1.0$ ). Actually, the integral timescale of atmospheric turbulence is on the order of $\sim 10$ min (Durán et al., 2011). The wind variations over timescales smaller than $\sim 10 \mathrm{~min}$ are attributed to turbulence, while variations over timescales larger than $\sim 10 \mathrm{~min}$ are attributed to meteorological effects. In general, the aeolian transport and wind
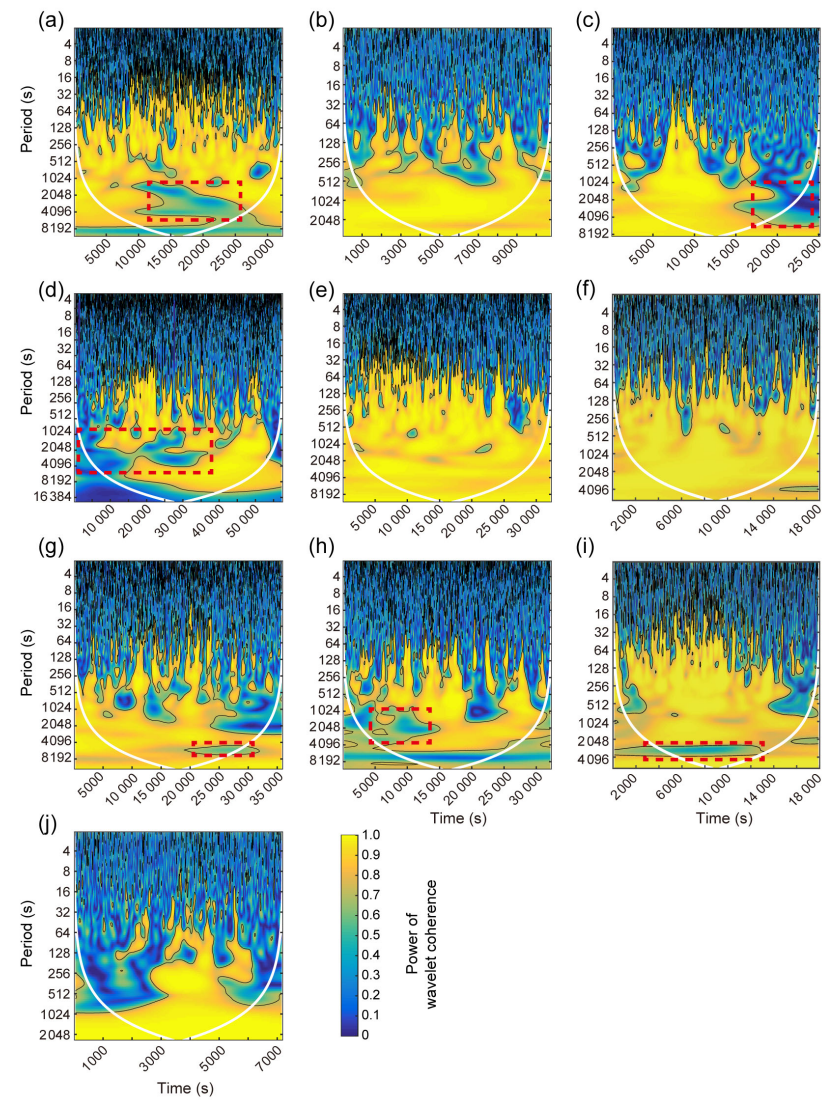

Figure 3. Coherence analyses between the space charge density and dust concentration. (a-j) Squared wavelet coherences, in turn, correspond to dust storms nos. 1-10 presented in Table 1 . The $5 \%$ significance level, estimated by Monte Carlo methods (Grinsted et al., 2004), against red noise is shown as black contour lines. The cone of influence where edge effects might distort the signal is shown as a white line. The relative phase relationship between the two time series is shown in Figs. S9-S13. Dashed rectangular boxes denote the destructions of large-scale electrification equilibrium at some time.

strength are highly correlated over timescales larger than $\sim 10 \mathrm{~min}$. Since the fine dust particles often follow the wind strictly, the large timescale strong correlation between $\rho$ and $M_{10}$ are certainly reasonable where the effects of turbulent fluctuations are excluded. In addition, the behavior of such significant large-timescale correlation (as indicated by the yellow area) is quite different from the observed dust storms (Fig. 3). That is, over 10 min timescales (period larger than 10 min in Fig. 3), the significant correlation is present in the whole time of Fig. 3b, e, f, $g$ and $j$ but is locally distributed in the time of Fig. 3a, c, d, h, and i.

To quantify the strong large timescale correlations between $\rho$ and $M_{10}$, we performed SLR analysis between the 10 min moving average (See Fig. S6 in the Supplement) of the $\rho$ and $M_{10}$ time series, where the fitted linear regression slope is equal to the $\mu^{*}$. The SLR analysis was performed 
Table 1. Overview of the 10 dust storms observed. $\rho_{\max }, \mu_{\max }^{*}, E_{z, \max }$ and $M_{10 \text {,max }}$ denote the maximum value of estimated space charge density, scaled charge-to-mass ratio, intensity of vertical E-field and $\mathrm{PM}_{10}$ mass concentration during a dust storm; $T_{\mathrm{a}}$ and $\mathrm{RH}$ denote the range of ambient temperature and relative humidity during a dust storm; $V_{\mathrm{b}, \text { min }}$ denotes the minimum visibility during a dust storm.

\begin{tabular}{|c|c|c|c|c|c|c|c|c|}
\hline $\begin{array}{l}\text { No. of } \\
\text { dust } \\
\text { storms }\end{array}$ & $\begin{array}{l}\text { Period } \\
(\mathrm{UTC}+8)\end{array}$ & $\begin{array}{r}\rho_{\max } \\
\left(\mathrm{C} \mathrm{m}^{-3}\right)\end{array}$ & $\begin{array}{r}\mu_{\max }^{*} \\
\left(\mathrm{C} \mathrm{kg}^{-1}\right)\end{array}$ & $\begin{array}{r}E_{z, \max } \\
\left(\mathrm{kV} \mathrm{m}^{-1}\right)\end{array}$ & $\begin{array}{c}M_{10, \max } \\
\left(\times 10^{-6}\right. \\
\left.\mathrm{kg} \mathrm{m}^{-3}\right)\end{array}$ & $\begin{array}{r}T_{\mathrm{a}} \\
\left({ }^{\circ} \mathrm{C}\right)\end{array}$ & $\begin{array}{l}\mathrm{RH} \\
(\%)\end{array}$ & $\begin{array}{r}V_{\mathrm{b}, \min } \\
(\mathrm{km})\end{array}$ \\
\hline 01 & 17 Apr 2017, 10:00-19:00 & $0.43 \times 10^{-6}$ & 0.41 & 94.3 & 2.77 & $16.9-21.6$ & $16.4-23.8$ & 0.09 \\
\hline 02 & 18 Apr $2017,15: 00-18: 00$ & $0.71 \times 10^{-7}$ & 0.47 & 14.7 & 0.19 & $17.1-18.9$ & $32.7-37.9$ & 0.55 \\
\hline 03 & 18 Apr 2017, 22:00-19 Apr 2017/05:00 & $0.11 \times 10^{-6}$ & 0.92 & 23.8 & 0.36 & $9.7-15.1$ & $12.7-43.1$ & 0.41 \\
\hline 04 & 19 Apr 2017, 06:00-22:00 & $0.10 \times 10^{-6}$ & 0.87 & 23.9 & 0.25 & $8.8-17.2$ & $8.1-47.4$ & 0.78 \\
\hline 05 & 20 Apr 2017, 08:00-17:00 & $0.18 \times 10^{-6}$ & 0.49 & 43.4 & 0.72 & $6.4-13.8$ & $10.9-22.1$ & 0.34 \\
\hline 06 & 22 Apr $2017,12: 00-17: 00$ & $0.38 \times 10^{-6}$ & 2.15 & 63.3 & 0.17 & $21.2-23.7$ & $4.2-6.2$ & 0.57 \\
\hline 07 & 3 May 2017, 08:00-18:00 & $0.23 \times 10^{-6}$ & 0.89 & 40.6 & 0.99 & $10.5-16.8$ & $7.7-53.1$ & 0.24 \\
\hline 08 & 10 May $2017,10: 00-19: 00$ & $0.83 \times 10^{-7}$ & 1.1 & 28.2 & 0.08 & $22.3-26.5$ & $7.1-10.2$ & 0.93 \\
\hline 09 & 12 May $2017,11: 00-16: 00$ & $0.44 \times 10^{-6}$ & 2.68 & 71.2 & 0.18 & $28.1-31.2$ & $3.4-6.4$ & 0.49 \\
\hline 10 & 20 May 2017, 23:00-21 May 2017/01:00 & $0.27 \times 10^{-6}$ & 0.69 & 20.1 & 0.39 & $21.4-23.7$ & $18.4-21.9$ & 0.55 \\
\hline
\end{tabular}

for a set of given temperature and $\mathrm{RH}$ intervals (within $2^{\circ} \mathrm{C}$ and $2 \%$ ). As shown in Fig. 4, there is a significant linear relationship between $\rho$ and $M_{10}$ at a given ambient temperature and RH (with median $R^{2}$ of $\sim 0.71-0.98$ and $p<0.01$; see Fig. S7 and Table S1 in the Supplement), suggesting that $\mu^{*}$ is nearly constant during a period that ambient temperature and RH are fixed. The long period constant $\mu^{*}$ implies that electrification equilibrium has been established (on average) where the rates of gain and loss of electrical charge are equal. $\mu^{*}$ is significantly influenced by environmental factors but independent of the particles' collisional dynamics and wind speed. For example, in Fig. $4, \mu^{*}$ (i.e., slope) varies from 0.264 to $0.421 \mathrm{C} \mathrm{kg}^{-1}$ for different ambient conditions but is constant at a given ambient condition, even though the wind speed changes dramatically.

\subsection{Temperature and RH dependence of $\mu^{*}$}

As shown in Fig. 5a, we find that $\mu^{*}$ varies widely from 0 to $3 \mathrm{C} \mathrm{kg}^{-1}$ for different ambient temperatures and RHs, consistent with the previous finding of the significant environmental dependence of granular electrification (Lacks and Sankaran, 2011; McCarty and Whitesides, 2008). According to the SLR analysis between $\rho$ and $M_{10}$, we used an MLR model to quantify the ambient temperature and RH dependence of $\mu^{*}$. The results of estimating coefficients, $F$ test for testing the model, and $t$ test for testing coefficients are shown in Table S2. It is seen from Fig. 5a that the MLR model is in good agreement with the measurement-based data $\left(R^{2}=\sim 0.71, p<0.001\right)$. Therefore, the dependence of $\mu^{*}$ on $T_{\mathrm{a}}$ and $\mathrm{RH}$ can be approximately described as

$$
\begin{aligned}
\mu^{*} & =\left(26955-2719 T_{\mathrm{a}}-698 T_{\mathrm{a}} \mathrm{RH}+89 T_{\mathrm{a}}^{2}\right. \\
& \left.+60950 \mathrm{RH}^{2}+24 T_{\mathrm{a}} \mathrm{RH}\right) \times 10^{-4}
\end{aligned}
$$

Clearly, the effects of ambient temperature and $\mathrm{RH}$ on the saturation $\mu^{*}$ are coupled and behave quite differently at different conditions (Fig. $5 \mathrm{~b}$ and c), as predicted by the MLR model. For example, $\mu^{*}$ increases (decreases) with increasing RH at $T_{\mathrm{a}}=27.5^{\circ} \mathrm{C}\left(5.5^{\circ} \mathrm{C}\right)$, while it decreases first and then increases at $\mathrm{T}_{\mathrm{a}}=16.5^{\circ} \mathrm{C}$, as shown in Fig. 5b. For various RH $(8.5 \%, 25.5 \%$ and $42.5 \%), \mu^{*}$ showed a similar pattern with increasing temperature: $\mu^{*}$ first decreased and then exhibited an upward trend, as shown in Fig. 5c.

\section{Discussion}

\subsection{Methods for estimating space charge density}

In granular materials, the electrification of dust particles is generally evaluated by the surface charge density and the charge-to-mass ratio (Merrison, 2012; Schmidt et al., 1998). The former is defined as the charge on a particle divided by its surface area, and the latter is defined as the charge on a particle divided by its mass. For laboratory experiments, the mean charge-to-mass ratio and even the surface charge density distribution on an individual particle can be accurately determined using optical microscopy such as particle image velocimetry (PIV; see Waitukaitis and Jaeger, 2013; Waitukaitis et al., 2014) and Kelvin force microscopy (KFM; see Baytekin et al., 2011b). However, such methods based on optical microscopy are difficult to use in field observations due to very complex environmental conditions (Harrison et al., 2016; Merrison, 2012). In this study, as in the method investigating the electrical effects of thunderstorms, clouds and aerosol layers in the atmosphere (e.g., MacGorman and Rust, 1998; Nicoll, 2012; Stolzenburg and Marshall, 1994; Zhou and Tinsley, 2007), the mean space charge density, which is the quantity of charge per unit volume, was determined indirectly. According to Gauss's law, the total space charge density is directly proportional to the divergence of 
(a)

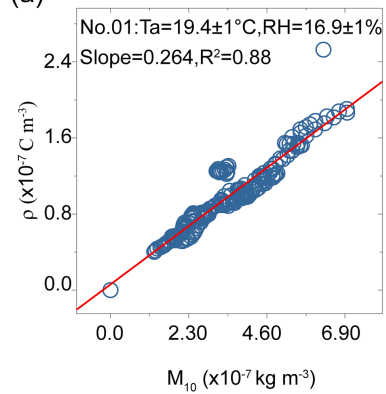

(c)

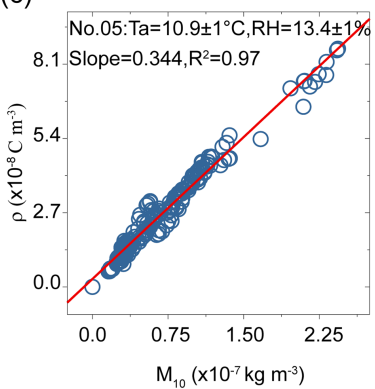

(e)

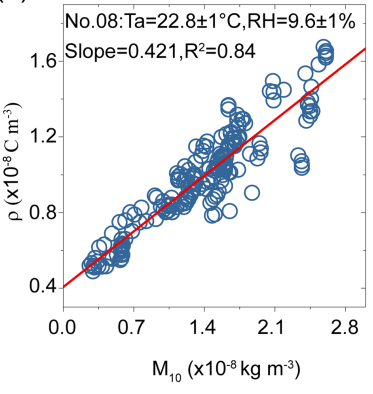

(b)

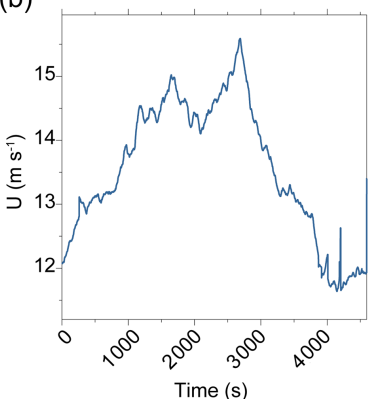

(d)

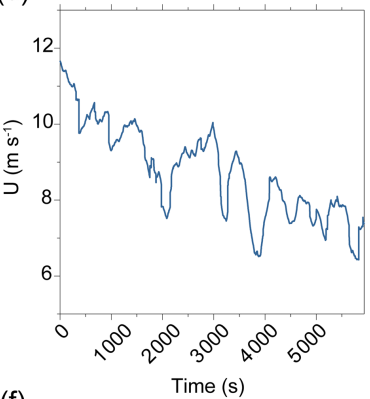

(f)

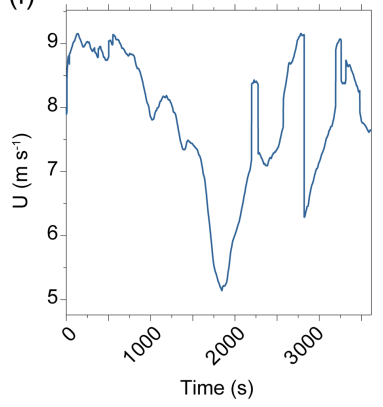

Figure 4. Significant linear relationships between the $10 \mathrm{~min}$ moving average of space charge density and dust concentration at the given ambient temperature and RH. In panels (a), (c) and (e), circles denote the data obtained from observations, and straight lines denote the linear regression. The slopes corresponds to the scaled charge-to-mass ratios $\mu^{*}$. Panels (b), (d) and (f) show wind speed during the corresponding period of (a), (c) and (e).

the E-fields. The VREFMs spacing is respectively $\sim 1.6,5$ and $10 \mathrm{~m}$ in the vertical, spanwise and streamwise directions owing to the rapid variation of E-fields along the vertical direction and slow variation along the spanwise and streamwise directions (see Fig. S5 in the Supplement), in order to eliminate the disturbances from other VREFMs and the observation tower, and thus the calculated space charge density is actually a measure of the mean charge of dust particles in a multi-cubic meter volume. As shown in Fig. 4, the calculated space charge density is $\sim 10^{-8}-10^{-7} \mathrm{C} \mathrm{m}^{-3}$ corresponding to $\sim 0.624 \times 10^{5}-10^{6} \mathrm{el} \mathrm{m}^{-3}$ (el denotes elementary charge), in accordance with the order of $0.7 \times 10^{6} \mathrm{el} \mathrm{m}^{-3}$ in a New Mexico dust devil (Crozier, 1964).

Previous measurements have demonstrated that the charge structure of dust clouds in dust storms could appear as unipo-

lar, bipolar and even multipolar. For example, Williams et al. (2009) measured the vertical E-field in dust storms and found both upward- and downward-pointing vertical E-field. They inferred that the dust cloud is unipolar if the nearground particle charge transfer is dominant, while the dust cloud is bipolar if upper-air (volume) charge transfer is dominant. Direct dust storm charge measurements by Kamra (1972) have also observed both positive and negative space charge at $1.25 \mathrm{~m}$ height above the ground. Additionally, our recent dust storm E-field measurements up to a height of $30 \mathrm{~m}$ have shown that dust cloud could be multipolar (Zhang et al., 2017). In this study, the derived space charge density at $5 \mathrm{~m}$ height is positive, which is certainly reasonable, although many studies have observed a negative space charge. In fact, the charge structure of dust storms is closely associated with the transport of dust particles. There is no doubt that the large-scale and very-large-scale motions of flow exist in the high Reynolds number atmospheric surface layer (Hutchins et al., 2012), affecting the transport of dust particles because of dust following wind flow exactly (Jacob and Anderson, 2016). We can expect that a bipolar charge structure in each large-scale motions is produced by the bidisperse suspensions of oppositely charged particles (Renzo and Urzay, 2018). Consequently, the multipolar charge structure of dust storms is formed by a series of bipolar charge of large-scale motions.

It is worth noting that E-fields as high as $\sim 181 \mathrm{kV} \mathrm{m}^{-1}$ are rarely found at a few meters height in the literature (except within the dust devils where horizontal E-fields exceeded $100 \mathrm{kV} \mathrm{m}^{-1}$; see Jackson and Farrell, 2006), because the charging processes between granular materials are very sensitive to the properties and size distribution of grains (Houghton et al., 2013). In our study, the saline-alkali soil at the QLOA site may facilitate the charge separation of dust particles, which may lead to high E-field intensity in dust storms.

\subsection{The physical mechanisms for electrification equilibrium}

Previous granular studies (e.g., Harper and Dufek, 2016; Matsuyama and Yamamoto, 1995) have shown that the individual particles in millimeter-sized granular flows were able to acquire a certain amount of saturation or equilibrium charge, where no further charge transfer occurs in spite of subsequent impacts between particles. The physical mechanisms for electrification saturation of individual particles can be attributed to the dielectric breakdown of air (Harper and Dufek, 2016; Matsuyama and Yamamoto, 1995; McCarty et al., 2007) and a repelling E-field in the charge transfer direction (Castle and Schein, 1995). More recently, a study of the contact electrification of micron-sized silica particles have also demonstrated that the surface charge density of the charged particles closely distributed around a constant value of $0.02 \mathrm{mC} \mathrm{m}^{-2}$ (that is, particles having a saturation 

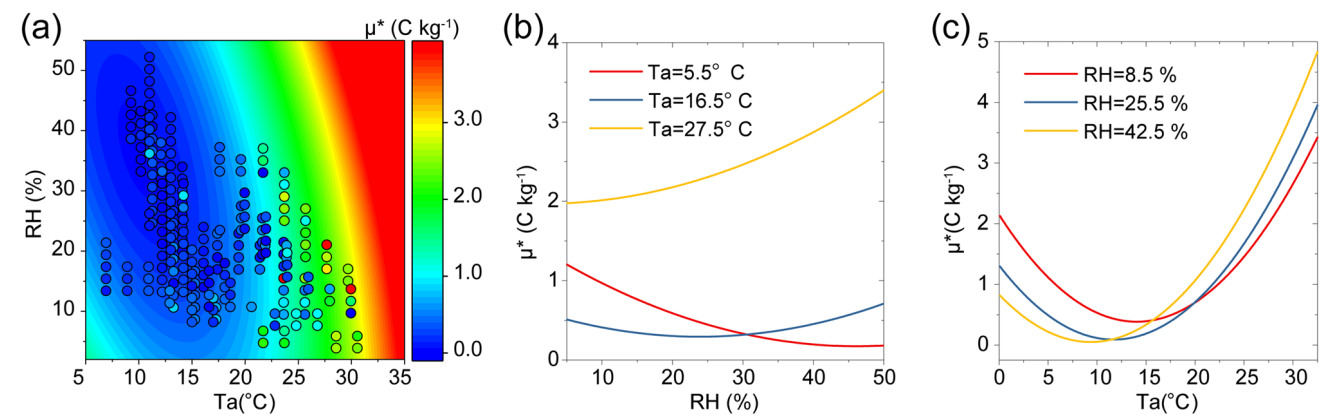

Figure 5. Dependence of the $\mu^{*}$ on environmental factors. (a) Comparison of the MLR model (contour) with measurement-based data (circle). (b) The predicted $\mu^{*}$ as a function of RH for different ambient temperatures. (c) The predicted $\mu^{*}$ as a function of ambient temperature for different RHs.

or equilibrium charge), which is most possibly caused by the alternative mechanisms such as electron field emission and charge spreading at the particle contact site (Alois et al., 2017). In the present study, the large-scale electrification equilibrium effects widely exist in dust storms (Figs. 3 and 4). However, in dust storms, we propose that such electrification equilibrium of a large-scale system (averaged over multi-cubic meter volume and $10 \mathrm{~min}$ ) is a dynamic equilibrium rather than the saturation of individual particles. In this case, the charges on dust particles transfer between the large-scale systems at an equal rate, meaning there is no net charge exchange. Charge transfer between individual dust particles may in fact occur, but to such an extent that we cannot observe the changes in $\mu^{*}$ of the large-scale system under certain ambient condition. It should be emphasized that the concept of large-scale electrification equilibrium is only applied to the dust storms under certain ambient condition; that is, $\mu^{*}$ is constant with varying particles' dynamics at given temperature and RH. Once ambient temperature or RH is changed, the large-scale system will reach a new electrification equilibrium. Consequently, such equilibrium can be termed environmental-dependent equilibrium effects. The equilibrium state is occasionally broken down (characterized by a weak large-scale correlation between space charge density and dust concentration) in several dust storms (e.g., Fig. 3c and d, as well as little $R^{2}$ in Fig. S7) and the reason for this is unclear. Further research is needed to explore the mechanisms leading to the occasional absence of significant large-timescale correlations.

In addition, the equilibrium value $\left(\mu^{*}\right)$ of the large-scale system was found to be strongly influenced by RH and ambient temperature in dust storms during our field observations. While water is not necessary for contact electrification (Baytekin et al., 2011a), a variety of studies indicated that such charge separation was strongly dependent on the RH (Esposito et al., 2016; McCarty and Whitesides, 2008; Xie and Han, 2012; Alois et al., 2018; Zhang et al., 2017). The proposed reasons for this are twofold: on one hand, the presence of adsorbed water could increase surface conduc- tivity and particle-particle effective contact area, thus facilitating the ion or electron transfer (McCarty and Whitesides, 2008; Alois et al., 2018); on the other hand, $\mathrm{OH}^{-}$ions in adsorbed surface water could also act as charge carrier $(\mathrm{Gu}$ et al., 2013; Lacks and Sankaran, 2011; McCarty and Whitesides, 2008). We also found that $\mu^{*}$ was strongly affected by the ambient temperature. This is consistent with other reports, which showed that the dielectric constant and conductivity of the adsorbed water were significantly linked to the ambient temperature (Gu et al., 2013; Lacks and Sankaran, 2011; Wei and Gu, 2015). As shown in Fig. 5b and c, the predicted $\mu^{*}$ is nonlinearly related to ambient temperature and RH. Specifically, the predicted $\mu^{*}$ increases at $T_{\mathrm{a}}=27.5^{\circ} \mathrm{C}$ but decreases at $T_{\mathrm{a}}=5.5^{\circ} \mathrm{C}$ with increasing $\mathrm{RH}$. This result has also been verified by other studies (Xie and Han, 2012; Zheng et al., 2014). For example, by considering the effects of a water film on the particle-particle effective contact area, Zheng et al. (2014) revealed that the net charge transfer between two particles increased first then decreased with increasing RH. In addition, a wind-tunnel measurement found that the E-fields produced by charged sand particles increased first then decreased with increasing ambient temperature when $\mathrm{RH}=17 \%$ (Xie and Han, 2012). Therefore, in Fig. $5 \mathrm{~b}$, the different patterns of $\mu^{*}$ at different ambient temperatures could be explained by the coupling effects between the nonlinear affecting factors ambient temperature and $\mathrm{RH}$.

\subsection{Implications for quantifying electrostatics in dust storms}

Dust electrification is very complex due to particle-particle interactions (a non-equilibrium charging process involving a wide range of length- and timescales) and particleturbulence interactions (particle inertia varying over several orders of magnitude at high Reynolds-number flow in dust storms). To quantitatively predict the charge transfer processes between dust particle collisions/contacts, a large number of theoretical models have been developed based on different physical mechanisms, such as asymmetric contact $(\mathrm{Hu}$ 
et al., 2012; Kok and Lacks, 2009; Kok and Renno, 2008), polarization by external E-fields (Pähtz et al., 2012), statistical variations of material properties (Apodaca et al., 2010) and shift of aqueous ions (Gu et al., 2013). Moreover, the charge carriers among them could be electrons (Kok and Lacks, 2009), ions (McCarty and Whitesides, 2008), trapped holes (Hu et al., 2012) and bits of nanoscopic material (Apodaca et al., 2010), depending on the material properties and condition involved (Lacks and Sankaran, 2011; Wei and $\mathrm{Gu}, 2015)$. These models mainly focus on explaining sizedependent charging (that is, smaller particles tend to acquire a net negative charge during collisions with larger particles) of dust particles without considering the environmental factors, which have been found to considerably affect dust electrification. Currently, the existing electrification models cannot explicitly account for the effects of environmental factors RH and ambient temperature (Harrison et al., 2016; Lacks and Sankaran, 2011; Wei and Gu, 2015) and thus field observations are the primary way for exploring dust electrification.

Dust storms act as a natural experiment through which the granular electrification effects can be evaluated. We found that space charge density and dust concentration were significantly correlated over large timescales. This is clear observational evidence of the large-scale electrification equilibrium effects in dust storms. This finding has potentially important implications for exploring the role of electrical effects in dust events. First, the electrification equilibrium will be helpful in quantifying the electrical effects during dust storms. The fact that the charge transfer process depends strongly on particle dynamics has been previously demonstrated $\mathrm{Hu}$ et al., 2012; Kok and Lacks, 2009; Wei and Gu, 2015). To quantify the particle charges (and therefore electrostatic forces) exactly, tracking the history of particle dynamics is required. However, it is too complex to be determined due to the polydisperse two-phase flow (dust storms) at very large Reynolds numbers of up to $R e_{\tau} \approx 4 \times 10^{6}$ (Wang and Zheng, 2016). Our results suggest that particle dynamics can be excluded reasonably well in the future quantification of electrical effects. Second, the theoretical understanding of charging electrification is expected to be facilitated by the equilibrium effects. As mentioned previously, the underlying physical mechanism behind dust electrification is extremely complex because such electrification is a non-equilibrium phenomenon that involves a wide range of length- and timescales (Lacks and Sankaran, 2011; Wei and Gu, 2015). The presence of electrification equilibrium in dust storms allows us to investigate the equilibrium mechanism, in contrast to the dynamic charging mechanism, which is believed to be straightforward.

\section{Conclusions}

One of the most important and difficult issues in investigating the wind-erosion phenomenon is the accurate quantifi- cation of the dust and sand electrification. As noted previously, electrical effects can considerably influence the dust transport and lifting processes. In this study, to evaluate the electrical properties of airborne dust particles in dust storms, we performed continuous field measurements at QLOA site from 21 March to 2 June 2017.

Overall, we have successfully monitored 10 complete dust storms lasting a total of $\sim 66 \mathrm{~h}$. According to a series of extensive analyses, we found that the average space charge density and dust concentration were significantly correlated over 10 min timescales, providing an observational evidence for the large-scale (over $10 \mathrm{~min}$ and multi-cubic meter volume) electrification equilibrium effects in dust storms, even though such effects are absent occasionally. In addition, the equilibrium scaled charge-to-mass ratio $\mu^{*}$ is found to be independent of wind speed but is a nonlinear function of the ambient temperature and RH. Our findings have potentially important implications for exploring the role of electrical effects in dust events, especially in the aspect of the quantification of electrical effects in dust storms. In further E-fields model of dust storms, the charge-to-mass ratio of airborne dust particles can be considered as a constant if the ambient temperature and RH do not change dramatically.

Data availability. The experimental data that support the findings of this study are available in Zenodo data repository, https://doi.org/10.5281/zenodo.1188752 (Zhang and Zheng, 2018).

Supplement. The supplement related to this article is available online at: https://doi.org/10.5194/acp-18-17087-2018-supplement.

Author contributions. XZ designed the QLOA. HZ and XZ designed the study. HZ performed the field observations and data analyses as well as wrote the manuscript, which was guided and edited by XZ. All authors discussed the results and commented on the manuscript.

Competing interests. The authors declare that they have no conflict of interest.

Acknowledgements. We thank Haihua Gu, Xuebo Li, Ao Mei, Guowen Han, Yirui Liang, Guohua Wang, Hongyou Liu, and Wei Zhu for providing useful assistance in the field observations and also thank Youhe Zhou for helpful comments and discussions. We acknowledge support from the National Natural Science Foundation of China (no. 11490553) and the Young Elite Scientists Sponsorship Program by CAST (no. 2017QNRC001). Thanks are also extended to the editor, Paola Formenti, and to the reviewers, Jonathan Merrison and two anonymous reviewers, for their careful work and thoughtful suggestions that greatly improved the manuscript. 
Edited by: Paola Formenti

Reviewed by: Jonathan Merrison and two anonymous referees

\section{References}

Aizawa, K., Cimarelli, C., Alatorre-Ibargüengoitia, M., Yokoo, A., Dingwell, D., and Iguchi, M.: Physical properties of volcanic lightning: Constraints from magnetotelluric and video observations at Sakurajima volcano, Japan, Earth Planet. Sci. Lett., 444, 45-55, https://doi.org/10.1016/j.eps1.2016.03.024, 2016.

Alois, S., Merrison, J., Iversen, J. J., and Sesterhenn, J.: Contact electrification in aerosolized monodispersed silica microspheres quantified using laser based velocimetry, J. Aerosol Sci., 106, 110, https://doi.org/10.1016/j.jaerosci.2016.12.003, 2017.

Alois, S., Merrison, J., Iversen, J. J., and Sesterhenn, J.: Quantifying the contact electrification of aerosolized insulating particles, Powder Technol., 332, 106-113, https://doi.org/10.1016/j.powtec.2018.03.059, 2018.

Apodaca, M., Wesson, P., Bishop, K., Ratner, M., and Grzybowski, B.: Contact electrification between identical materials, Angew. Chem.-Int. Edit., 49, 946-949, https://doi.org/10.1002/anie.200905281, 2010.

Baytekin, H., Baytekin, B., Soh, S., and Grzybowski, B.: Is water necessary for contact electrification?, Angew. Chem.-Int. Edit., 50, 6766-6770, https://doi.org/10.1002/anie.201008051, 2011a.

Baytekin, H., Patashinski, A., Branicki, M., Baytekin, B., Soh, S., and Grzybowski, B.: The mosaic of surface charge in contact electrification, Science, 333, 308-312, https://doi.org/10.1126/science.1201512, 2011 b.

Bendat, J. and Piersol, A.: Random data: Analysis and measurement procedures, John Wiley Sons, Hoboken, NJ, 2011.

Bo, T., Zhang, H., and Zheng, X.: Charge-to-mass ratio of saltating particles in wind-blown sand, Sci. Rep., 4, 5590, https://doi.org/10.1038/srep05590, 2014.

Castle, G. and Schein, L.: General model of sphere-sphere insulator contact electrification, J. Electrostat., 36, 165-173, https://doi.org/10.1016/0304-3886(95)00043-7, 1995.

Crozier, W.: The electric field of a New Mexico dust devil, J. Geophys. Res., 69, 5427-5429, https://doi.org/10.1029/JZ069i024p05427, 1964

Durán, O., Claudin, P., and Andreotti, B.: On aeolian transport: Grain-scale interactions, dynamical mechanisms and scaling laws, Aeolian Res., 3, 243-270, https://doi.org/10.1016/j.aeolia.2011.07.006, 2011.

Esposito, F., Molinaro, R., Popa, C., Molfese, C., Cozzolino, F., Marty, L., Taj-Eddine, K., Achille, G., Franzese, G., and Silvestro, S.: The role of the atmospheric electric field in the dust-lifting process, Geophys. Res. Lett., 43, 5501-5508, https://doi.org/10.1002/2016GL068463, 2016.

Grinsted, A., Moore, J., and Jevrejeva, S.: Application of the cross wavelet transform and wavelet coherence to geophysical time series, Nonlinear Proc. Geoph., 11, 561-566, https://doi.org/10.5194/npg-11-561-2004, 2004.

Gu, Z., Wei, W., Su, J., and Yu, C.: The role of water content in triboelectric charging of wind-blown sand, Sci. Rep., 3, 1337, https://doi.org/10.1038/srep01337, 2013.
Harper, J. and Dufek, J.: The effects of dynamics on the triboelectrification of volcanic ash, J. Geophys. Res.-Atmos., 121, 82098228, https://doi.org/10.1002/2015JD024275, 2016.

Harper, J., Mcdonald, G., Dufek, J., Malaska, M., Burr, D., Hayes, A., Mcadams, J., and Wray, J.: Electrification of sand on Titan and its influence on sediment transport, Nat. Geosci., 10, 260 265, https://doi.org/10.1038/NGEO2921, 2017.

Harrison, R., Barth, E., Esposito, F., Merrison, J., Montmessin, F., Aplin, K., Borlina, C., Berthelier, J., Dprez, G., and Farrell, W.: Applications of electrified dust and dust devil electrodynamics to Martian atmospheric electricity, Space Sci. Rev., 203, 299-345, https://doi.org/10.1007/s11214-016-0241-8, 2016.

Holman, I., Rivas-Casado, M., Bloomfield, J., and Gurdak, J.: Identifying non-stationary groundwater level response to North Atlantic oceanatmosphere teleconnection patterns using wavelet coherence, Hydrogeol. J., 19, 1269-1278, https://doi.org/10.1007/s10040-011-0755-9, 2011.

Houghton, I., Aplin, K., and Nicoll, K.: Triboelectric charging of volcanic ash from the 2011 Grimsvotn eruption, Phys. Rev. Lett., 111, 118501, https://doi.org/10.1103/PhysRevLett.111.118501, 2013.

$\mathrm{Hu}, \mathrm{W} ., \mathrm{Xie}, \mathrm{L}$. , and Zheng, X.: Contact charging of silica glass particles in a single collision, Appl. Phys. Lett., 101, 3504-3615, https://doi.org/10.1063/1.4752458, 2012.

Hutchins, N., Chauhan, K., Marusic, I., Monty, J., and Klewicki, J.: Towards reconciling the large-scale structure of turbulent boundary layers in the atmosphere and laboratory, Bound.-Lay. Meteorol., 145, 273-306, https://doi.org/10.1007/s10546-012-9735-4, 2012.

Jackson, T. and Farrell, W.: Electrostatic fields in dust devils: an analog to Mars, IEEE T. Geosci. Remote, 44, 2942-2949, https://doi.org/10.1109/TGRS.2006.875785, 2006.

Jacob, C. and Anderson, W.: Conditionally averaged large-scale motions in the neutral atmospheric boundary layer: Insights for aeolian processes, Bound.-Lay. Meteorol., 162, 21-41, https://doi.org/10.1007/s10546-016-0183-4, 2016.

Kamra, A.: Measurements of the electrical properties of dust storms, J. Geophys. Res., 77, 5856-5869, https://doi.org/10.1029/JC077i030p05856, 1972.

Kok, J. and Lacks, D.: Electrification of granular systems of identical insulators, Phys. Rev. E, 79, 051304, https://doi.org/10.1103/PhysRevE.79.051304, 2009.

Kok, J. and Renno, N.: Electrostatics in windblown sand, Phys. Rev. Lett., 100, 014501, https://doi.org/10.1103/PhysRevLett.100.014501, 2008.

Lacks, D. and Sankaran, R.: Contact electrification of insulating materials, J. Phys. D-Appl. Phys., 44, 453001, https://doi.org/10.1088/0022-3727/44/45/453001, 2011.

MacGorman, D. and Rust, W.: The electrical nature of storms, Oxford University Press, New York, 1998.

Matsuyama, T. and Yamamoto, H.: Charge relaxation process dominates contact charging of a particle in atmospheric conditions, J. Phys. D-Appl. Phys., 28, 2418, https://doi.org/10.1088/00223727/28/12/005, 1995.

McCarty, L. and Whitesides, G.: Electrostatic charging due to separation of ions at interfaces: contact electrification of ionic electrets, Angew. Chem.-Int. Edit., 47, 2188, https://doi.org/10.1002/anie.200701812, 2008. 
McCarty, L., Winkleman, A., and Whitesides, G.: Ionic electrets: Electrostatic charging of surfaces by transferring mobile ions upon contact, J. Am. Chem. Soc., 129, 4075-4088, https://doi.org/10.1021/ja067301e, 2007.

Merrison, J.: Sand transport, erosion and granular electrification, Aeolian Res., 4, 1-16, https://doi.org/10.1016/j.aeolia.2011.12.003, 2012.

Nicoll, K.: Measurements of atmospheric electricity aloft, Surv. Geophys., 33, 991-1057, https://doi.org/10.1007/s10712-0129188-9, 2012

Pähtz, T., Herrmann, H., and Shinbrot, T.: Why do particle clouds generate electric charges? Nat. Phys., 6, 364-368, https://doi.org/10.1038/NPHYS1631, 2012.

Pollack, G. and Stump, D.: Electromagnetism, Addison Wesley, San Fransisco, 2002.

Renzo, M. D. and Urzay, J.: Aerodynamic generation of electric fields in turbulence laden with charged inertial particles, Nat. Commun., 9, 1676, https://doi.org/10.1038/s41467-018-039587, 2018.

Rudge, W.: Atmospheric electrification during South African dust storms, Nature, 91, 31-32, https://doi.org/10.1038/091031a0, 1913.

Schmidt, D., Schmidt, R., and Dent, J.: Electrostatic force on saltating sand, J. Geophys. Res.-Atmos., 103, 8997-9001, https://doi.org/10.1029/98JD00278, 1998.

Shao, Y.: Physics and modelling of wind erosion, Springer-Verlag, Netherlands, 2000.

Stolzenburg, M. and Marshall, T.: Testing models of thunderstorm charge distributions with Coulomb's law, J. Geophys. Res.Atmos., 99, 25921-25932, https://doi.org/10.1029/94JD02332, 1994.

Waitukaitis, S. and Jaeger, H.: In situ granular charge measurement by free-fall videography, Rev. Sci. Instrum., 84, 277, https://doi.org/10.1063/1.4789496, 2013.

Waitukaitis, S., Lee, V., Pierson, J., Forman, S., and Jaeger, H.: Size-dependent same-material tribocharging in insulating grains, Phys. Rev. Lett., 112, https://doi.org/10.1103/PhysRevLett.112.218001, 2014.

Wang, G. and Zheng, X.: Very large-scale motions in the atmospheric surface layer: a field investigation, J. Fluid Mech., 802, 464-489, https://doi.org/10.1017/jfm.2016.439, 2016.

Wang, Q., Dong, X., Fu, J. S., Xu, J., Deng, C., Jiang, Y., Fu, Q., Lin, Y., Huang, K., and Zhuang, G.: Environmentally dependent dust chemistry of a super Asian dust storm in March 2010: observation and simulation, Atmos. Chem. Phys., 18, 3505-3521, https://doi.org/10.5194/acp-18-3505-2018, 2018.

Wang, X., Edwards, R., Auler, A., Cheng, H., Kong, X., Wang, Y., Cruz, F., Dorale, J., and Chiang, H.: Hydroclimate changes across the Amazon lowlands over the past 45,000 years, Nature, 541, 204, https://doi.org/10.1038/nature20787, 2017.
Wei, W. and $\mathrm{Gu}, \mathrm{Z}$. E Electrification of particulate entrained fluid flows-mechanisms, applications, and numerical methodology, Phys. Rep., 600, 1-53, https://doi.org/10.1016/j.physrep.2015.10.001, 2015.

Williams, E., Nathou, N., Hicks, E., Pontikis, C., Russell, B., Miller, M., and Bartholomew, M. J.: The electrification of dust-lofting gust fronts ("haboobs") in the Sahel, Atmos. Res., 91, 292-298, https://doi.org/10.1016/j.atmosres.2008.05.017, 2009.

Wiles, J., Fialkowski, M., Radowski, M., Whitesides, G., and Grzybowski, B.: Effects of surface modification and moisture on the rates of charge transfer between metals and organic materials, J. Phys. Chem. B, 108, 20296-20302, https://doi.org/10.1021/jp0457904, 2007.

Xie, L. and Han, K.: Influence of relative humidity on the aeolian electric field, Aeolian Res., 7, 45-50, https://doi.org/10.1016/j.aeolia.2012.01.002, 2012.

Yair, Y., Katz, S., Yaniv, R., Ziv, B., and Price, C.: An electrified dust storm over the Negev desert, Israel, Atmos. Res., 181, 6371, https://doi.org/10.1016/j.atmosres.2016.06.011, 2016.

Zhang, H. and Zheng, X.: Quantifying the large-scale electrification equilibrium effects in dust storms using field observations at Qingtu Lake Observatory [Data set], Zenodo, https://doi.org/10.5281/zenodo.1188752, 2018.

Zhang, H., Zheng, X., and Bo, T.: Electrification of saltating particles in wind-blown sand: Experiment and theory, J. Geophys. Res.-Atmos., 118, 12086-12093, https://doi.org/10.1002/2013JD020239, 2013.

Zhang, H., Bo, T., and Zheng, X.: Evaluation of the electrical properties of dust storms by multi-parameter observations and theoretical calculations, Earth Planet. Sci. Lett., 461, 141-150, https://doi.org/10.1016/j.epsl.2017.01.001, 2017.

Zheng, X., Huang, N., and Zhou, Y.: Laboratory measurement of electrification of wind-blown sands and simulation of its effect on sand saltation movement, J. Geophys. Res.-Atmos., 108, 4322, https://doi.org/10.1029/2002JD002572, 2003.

Zheng, X., Zhang, R., and Huang, H.: Theoretical modeling of relative humidity on contact electrification of sand particles, Sci. Rep., 4, 4399, https://doi.org/10.1038/srep04399, 2014.

Zheng, X. J.: Electrification of wind-blown sand: recent advances and key issues, Eur. Phys. J. E, 36, 138, https://doi.org/10.1140/epje/i2013-13138-4, 2013.

Zhou, L. and Tinsley, B.: Production of space charge at the boundaries of layer clouds, J. Geophys. Res.-Atmos., 112, D11203, https://doi.org/10.1029/2006JD007998, 2007. 